\title{
Initiative der DRG zur strukturierten Befundung
}

Die strukturierte Befundung hat in den letzten Jahren zunehmend Einzug in die onkologische Diagnostik gehalten und wird von den zuweisenden Klinikern vermehrt in der täglichen Routinebefundung eingefordert. Aktuell werden onkologische Befundberichte in der klinischen Radiologie weitgehend als Freitexte in Prosa-Form verfasst, welche üblicherweise keiner festgelegten Struktur folgen und vom jeweiligen Radiologen individuell gestaltet werden können. Diese Freitextbefunde weisen nicht nur Einschränkungen hinsichtlich der Lesbarkeit und damit der zuverlässigen Erfassung wichtiger klinischer Befunde auf, sondern führen unter Umständen auch dazu, dass wichtige Informationen für klinische Entscheidungen, wie kurativer versus palliativen Therapieansatz, nicht suffizient erfasst werden.

In klinischen Studien konnte gezeigt werden, dass mittels der strukturierten Befundung $u$. a. eine deutlich verbesserte Befundbeschreibung und Kommunikation mit dem zuweisenden Onkologen und Chirurgen erreicht werden kann. So zeigte sich bei Brook et al. (Radiology 2015), dass Chirurgen beim Pankreas-Karzinom die für die OP-Planung klinisch relevanten Befundinhalte in normalen „Prosa“-Befundtexten nur in 25-43\% vorfanden, hingegen anhand von 69-98\% der strukturierten Befunde die OP-Entscheidung getroffen werden konnte. Diese Informationen sind aber gerade im Hinblick auf eine moderne und individuell angepasste Tumortherapie für den betreuenden Onkologen, Chirur- gen, interventionellen Radiologen und Strahlentherapeuten von enormer Wichtigkeit.

In Zusammenarbeit mit der AG Onkologische Bildgebung, AG Gastrointestinal- und Abdominaldiagnostik und der AG Informationstechnologie sowie klinischen Vertretern u. a. der Deutschen Gesellschaft für Allgemein- und Viszeralchirurgie wurden in einem Konsensus-Meeting am Uniklinikum Köln am 8.2.2017 strukturierte Befundvorlagen für das Rektum-Karzinom, Kolon-Karzinom und solide Pankreas-Karzinom entwickelt. Sie finden weitere Informationen auf der DRG-Homepage www.drg.de unter
News sowie zukünftig die Konsensusbasierten strukturierten Befundvorlagen unter www.befundung.drg.de

Wir möchten Sie herzlich dazu einladen mitzuentscheiden, wie der radiologische Befund von Morgen aussieht und würden uns über ihre aktive Mitarbeit sehr freuen!

\section{IHRE MEINUNG IST GEFRAGT!}

Nehmen Sie jetzt an der Online-Umfrage teil und entscheiden Sie mit über die Zukunft des radiologischen Befundes. 
Sollten Sie Fragen zur Initiative und zu den Umfragen haben, wenden Sie sich gerne an:

PD Dr. med. Thorsten Persigehl

-Leitender Oberarzt-

Institut für Diagnostische und Interventionelle Radiologie
Universitätsklinikum Köln

Kerpener Str. 62, 50937 Köln, Germany

++49/221/47896045

thorsten.persigehl@uk-koeln.de

Dr. med. Daniel Pinto dos Santos

- Oberarzt -
Institut für Diagnostische und Interventionelle Radiologie Universitätsklinikum Köln Kerpener Str. 62, 50937 Köln, Germany ++49/221/47896046

daniel.pinto-dos-santos@uk-koeln.de 